\title{
HUMAN-INDUCED VIBRATIONS ON FOOTBRIDGES. CURRENT CODES OF PRACTICE - CASE STUDY
}

\author{
KRUMKa KASAPOVA*, DOBRomir DINEV \\ University of Architecture, Civil Engineering and Geodesy; \\ 1 Smirnenski Blvd., 1046, Sofia, Bulgaria
}

[Received: 25 September 2019. Accepted: 03 February 2020]

doi: 10.7546/JTAM.50.20.03.05

\begin{abstract}
Although not vulnerable regarding structural capacity and integrity in most cases, lightweight structures may exhibit excessive vibration levels. When regarding public buildings or structures which may be exposed to the excitation of a significant number of people, for instance concert halls, grandstands, long-span floors, staircases in malls etc. the issue concerning vibrations induced by humans becomes an important part of the overall structural design process.

The current article explores the dynamic behaviour of Eeklo footbridge. Calculations of vertical acceleration levels according to the current codes are implemented. The result are summarized and analyzed in details.
\end{abstract}

KEY WORDS: Human-induced vibrations, vertical acceleration, footbridges

\section{INTRODUCTION}

As the structural designers strive toward more slender structures, the problem with the human-induced vibrations acquires great significance. The commonly used architectural practice of designing such structures as lightweight leads to inevitable decrease in the structural mass and stiffness, thus making the structures less inert and less resistant to the loads induced by humans. As a result, the structural vibrations may reach high, adverse for the occupants [1].

The current codes of practice, which are thoroughly analyzed in the present article through an existing structure - Eeklo footbridge, East Flanders, Belgium, adopt simplified procedures for the vibration serviceability assessment of footbridges. Their procedures are reduced to assuming a resonant load condition and therefore determining acceleration levels which are most probably enhanced.

A deficiency of the aforementioned regulations is that they disregard the humanhuman interaction, which may have a substantial effect on the maximum acceleration levels of the structure under consideration [2]. Current paper should be considered as integral part of [3] as the theoretical background of the regulations is thereof exposed.

\footnotetext{
${ }^{*}$ Corresponding author e-mail: krumka_kasapova@abv.bg
} 


\section{Modal Analysis - EeKlo Footbridge}

The present article considers the slender Eeklo footbridge (Figure 1) as a running example. A thorough analysis of the dynamic behaviour of the bridge is performed throughout this work.

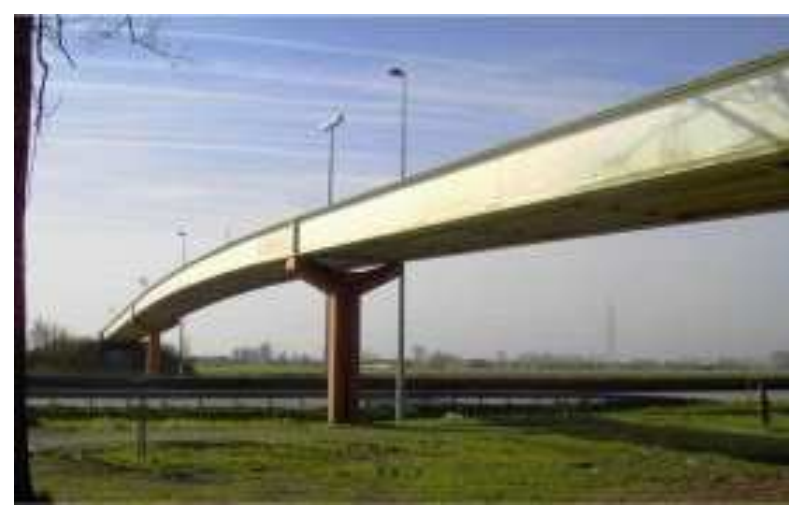

Fig. 1: Eeklo footbridge

Eeklo footbridge is situated in Eeklo, East Flanders (Belgium). The footbridge is used by pedestrians and cyclists to cross a highway. The structural model of the footbridge is a continuous beam with three spans and overall length of $96 \mathrm{~m}$. The central span has length of $42 \mathrm{~m}$, whereas the two side-ward spans $-27 \mathrm{~m}$. The structure consists of two main double-T steel girders with height $1.20 \mathrm{~m}$ and axial distance between them $3.4 \mathrm{~m}$, using the girders with their height as railings. T-shaped secondary beams are implemented parallel to the main girders, spaced approximately through $0.85 \mathrm{~m}$. There are transverse beams of same shape implemented through $4.20 \mathrm{~m}$ in the midspan and through $4.50 \mathrm{~m}$ in the side-ward spans. The bridge deck is implemented by steel sheets of thickness $8 \mathrm{~mm}$. The weight of the superstructure is $440 \mathrm{kN}$.

Modal analysis is executed for 10 mode shapes. Table 1 summarizes the results from the conducted modal analysis. Four of the natural modes of vibrations - from $f_{2}$ to $f_{5}$ - do not exceed $5.0 \mathrm{~Hz}$ (Figure 3), and therefore are recognized as modes that can be excited by human motion in compliance with [4-7]. Mode $2\left(f_{2}=1.71 \mathrm{~Hz}\right)$, which is a torsional mode of vibration, is characterized by a significant damping ratio $-\xi_{2}=1.94 \%$. This can be justified with the specificity of the structure - the side abutments and the intermediate pillars would have significant contribution to the dissipation of the energy. Similar reasoning may be applied for modes of interest 4 and 5, which are as well torsional modes and have high damping ratios (respectively $\xi_{4}=1.45 \%$ and $\xi_{5}=2.97 \%$ ). Regarding Mode 3 which is a bending mode, it differs 


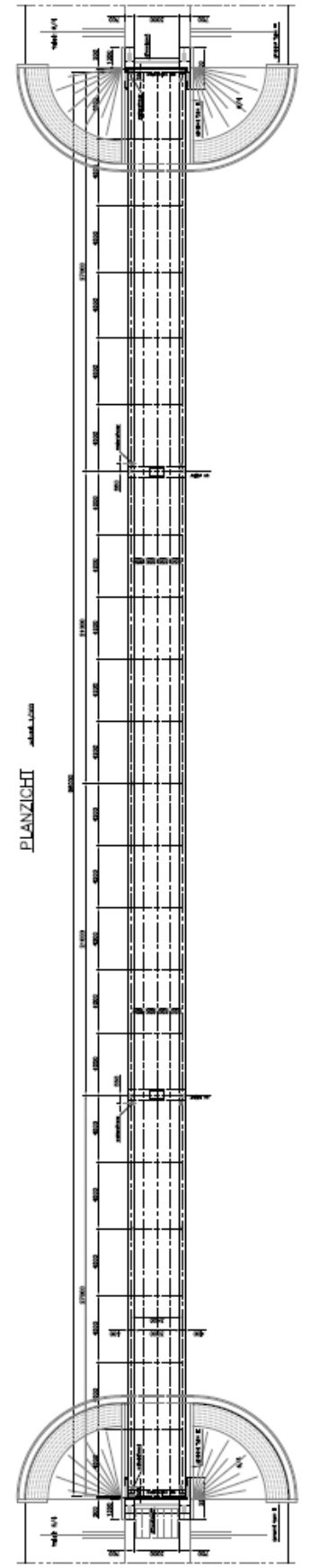

(a) Plan

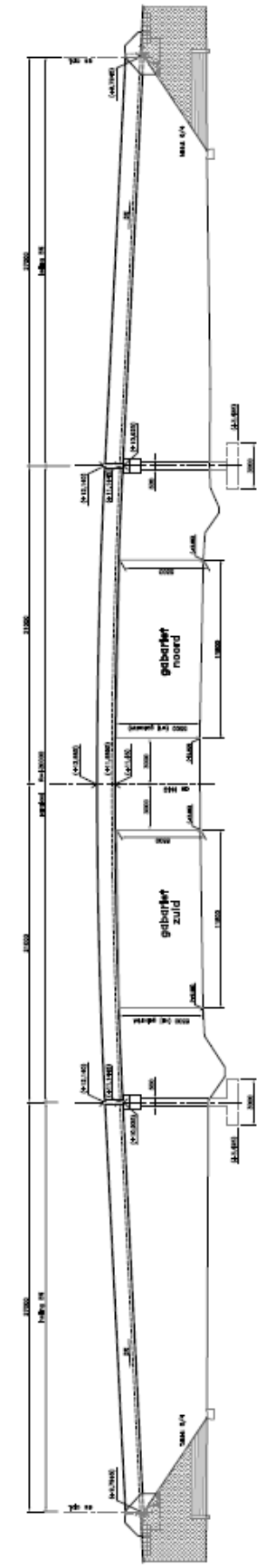

(b) Side view

Fig. 2: Plan (left) and side view (right) of the footbridge 

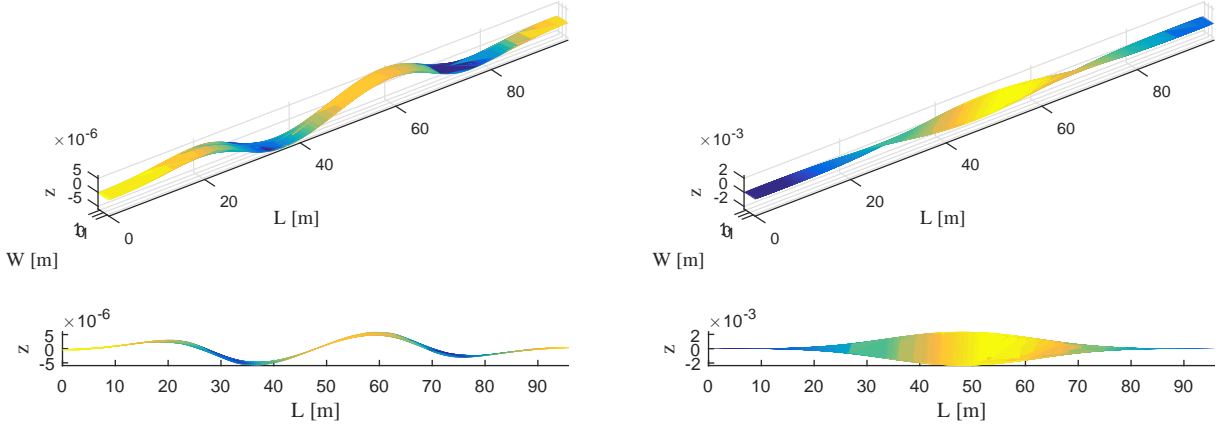

(a) Mode 1: $f_{1}=1.03 \mathrm{~Hz}$

(b) Mode 2: $f_{2}=1.70 \mathrm{~Hz}, \xi_{2}=1.94 \%$
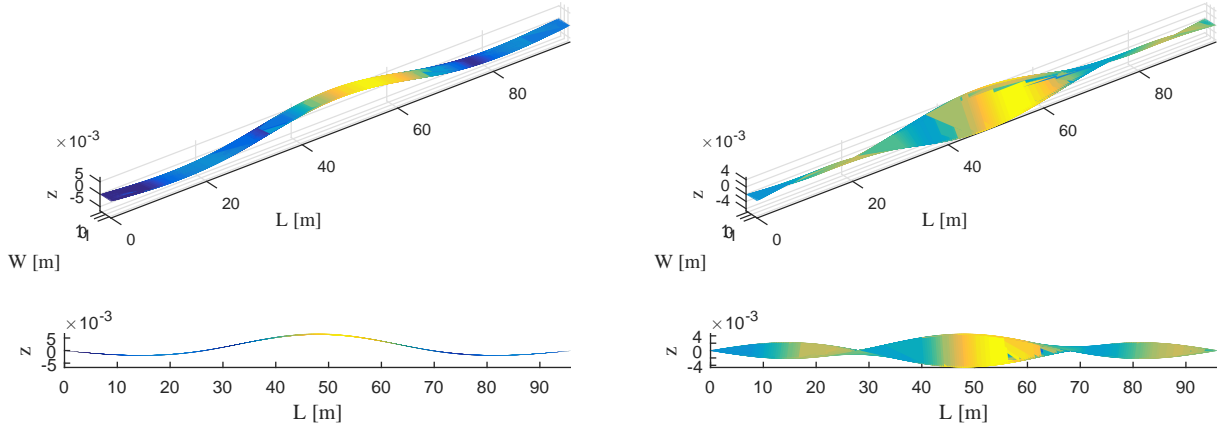

(c) Mode 3: $f_{3}=3.00 \mathrm{~Hz}, \xi_{3}=0.19 \%$

(d) Mode 4: $f_{4}=3.30 \mathrm{~Hz}, \xi_{4}=1.45 \%$

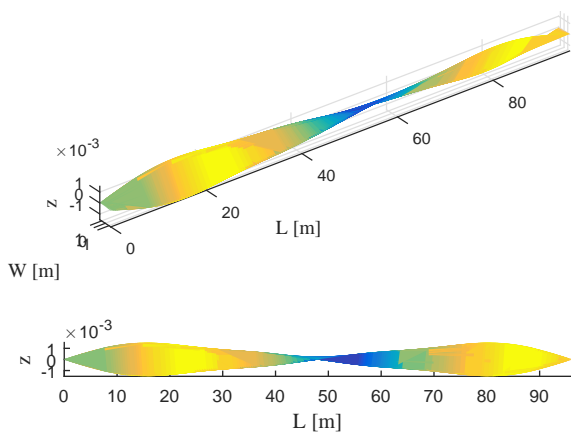

(e) Mode 5: $f_{5}=3.43 \mathrm{~Hz}, \xi_{5}=2.97 \%$

Fig. 3: Natural modes of vibrations for mode 1 up to mode 5

with appreciably smaller damping ratio $-\xi_{3}=0.19 \%$, which can be explained by the fact that as only obstruction of the free vibrations in this mode may be recognized the 
Table 1: Natural frequencies in $\mathrm{Hz}$ for different crowd densities and percentile difference $\Delta_{f}$ with respect to the empty footbridge

\begin{tabular}{ccccccccccccccc}
\hline \multicolumn{10}{c}{$d=15$ peds. $d=0.2$ ped. $/ \mathrm{m}^{2} d=0.5$ ped. $/ \mathrm{m}^{2} d=0.8$ ped. $/ \mathrm{m}^{2} d=1.0$ ped. $/ \mathrm{m}^{2} d=1.5$ ped. $/ \mathrm{m}^{2}$} \\
\hline Mode & $f$ & $f_{15}$ & $\Delta_{f}$ & $f_{0.2}$ & $\Delta_{f}$ & $f_{0.5}$ & $\Delta_{f}$ & $f_{0.8}$ & $\Delta_{f}$ & $f_{1.0}$ & $\Delta_{f}$ & $f_{1.5}$ & $\Delta_{f}$ \\
\hline 1 & 1.04 & 1.03 & 0.57 & 1.01 & 2.12 & 0.98 & 5.07 & 0.96 & 7.77 & 0.94 & 9.45 & 0.9 & 13.26 \\
2 & 1.71 & 1.7 & 0.46 & 1.68 & 1.73 & 1.64 & 4.16 & 1.6 & 6.44 & 1.58 & 7.87 & 1.52 & 11.18 \\
3 & 3.02 & 3 & 0.62 & 2.95 & 2.3 & 2.85 & 5.49 & 2.76 & 8.38 & 2.71 & 10.17 & 2.59 & 14.23 \\
4 & 3.30 & 3.29 & 0.32 & 3.26 & 1.21 & 3.21 & 2.94 & 3.15 & 4.58 & 3.12 & 5.62 & 3.04 & 8.07 \\
5 & 3.43 & 3.42 & 0.46 & 3.37 & 1.72 & 3.29 & 4.16 & 3.21 & 6.43 & 3.16 & 7.87 & 3.05 & 11.21 \\
6 & 5.75 & 5.72 & 0.65 & 5.61 & 2.44 & 5.42 & 5.81 & 5.24 & 8.87 & 5.14 & 10.76 & 4.89 & 15.03 \\
7 & 5.80 & 5.78 & 0.26 & 5.74 & 0.99 & 5.66 & 2.43 & 5.58 & 3.8 & 5.52 & 4.69 & 5.4 & 6.8 \\
8 & 6.10 & 6.07 & 0.39 & 6.01 & 1.46 & 5.88 & 3.59 & 5.75 & 5.63 & 5.67 & 6.94 & 5.48 & 10.14 \\
9 & 6.47 & 6.43 & 0.66 & 6.31 & 2.49 & 6.09 & 5.92 & 5.88 & 9.04 & 5.76 & 10.97 & 5.48 & 15.25 \\
10 & 6.94 & 6.91 & 0.47 & 6.82 & 1.73 & 6.66 & 4.09 & 6.51 & 6.19 & 6.42 & 7.48 & 6.23 & 10.35 \\
\hline
\end{tabular}

ability of the steel in the midspan to dissipate energy. Mode 1 is to be excluded from any further analysis as it is a longitudinal mode, not relevant due to the insignificant transversal modal displacements of the bridge deck.

In addition, Table 1 give information about the modified natural frequencies for all of the traffic classes considered in $[6,7]$ and the respective percentile differences with regard to the natural frequencies of the empty structure. The modification of

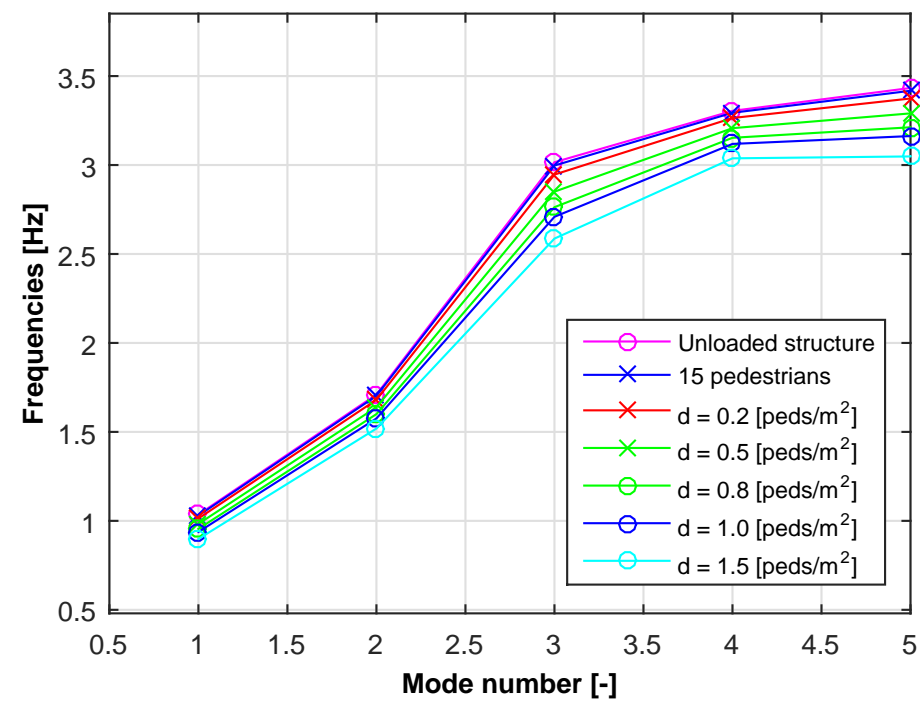

Fig. 4: Natural frequencies depending on the density of the crowd for the first five modes 
the natural frequencies is, obviously, principally perceptible for the higher crowd densities (Figure 4), where the influence of the added static mass of the pedestrians may exceed $10 \%$ (for crowd densities $d=1.0$ ped. $/ \mathrm{m}^{2}$ and $d=1.5$ ped. $/ \mathrm{m}^{2}$ ).

\section{Guidelines and Current Codes of Practice}

Most of the current regulations have the following procedure:

- Defining the modal characteristics of the structure - modal (or generalised) masses, natural frequencies, modal damping ratios, mode shapes and all the relevant information that can be derived by performing the vital modal analysis

- Defining the crowd loading by employing simplified equivalent load model

- For the natural modes of interest (these which can be excited by the humaninduced dynamic loads) determining the maximum accelerations due to the equivalent load model

- Comparing the maximum accelerations with imposed limits in terms of the comfort of the occupants

In the following, a brief summary and comparison of the best-known methodologies for the serviceability assessment is exposed.

\subsection{CALCUlATION OF THE DYNAMIC RESPONSE}

The dynamic response of a structure submitted to loading by human motion is calculated with the premise of resonant condition according to Eq.(1).

$$
a_{\max , n}=\frac{p_{n}}{m_{n}} \frac{1}{2 \xi_{n}},
$$

wherein $p_{n}$ - generalized load; $m_{n}$ - generalized mass; $\xi_{n}$ - modal damping ratio.

For each mode, whose frequency falls within the critical range, calculations for obtaining the maximum vibration levels should be performed considering that the respective natural frequency coincide with the pacing rate of the pedestrians.

\subsection{CASE STUdy - EEKLO FOOTBRIDGE}

The present section summarizes the results for the vertical accelerations of the Eeklo footbridge. The modal characteristics of the footbridge obtained by in-situ measurements are used for the calculation of the maximum acceleration levels. 


\subsubsection{DYNAMIC RESPONSE CALCULATIONS ACCORDING TO EUROCODE 5 AND BS 5400}

Here, only the third mode is considered for dynamic investigation of the structure, as it is the only one bending mode falling within the relevant frequency range, namely $f \leq 5.00 \mathrm{~Hz}$. Table 2 shows the variables needed for the final evaluation as well as the obtained accelerations according to [4].

Table 2: Maximum accelerations according to [4], $\mathrm{m} / \mathrm{s}^{2}$

\begin{tabular}{cccccccc}
\hline mode & $f, \mathrm{~Hz}$ & $\xi$ & $a_{\text {vert }, 1}$ & $k_{\text {vert }}$ & $a_{\text {vert }, n=13}$ & $a_{\text {vert }, n=0.6 A}$ & $a_{\text {limit }}$ \\
\hline Mode 3 & 3.02 & 0.0019 & 0.58 & 0.79 & 1.37 & 18.2 & 0.7 \\
\hline
\end{tabular}

The results derived for the crowd case $(n=0.6 A)$ unrealistically exceeds the limiting value of $a_{\text {limit }}=0.7 \mathrm{~m} / \mathrm{s}^{2}$. This doubtfully stems from the fact the only reduction factor considered is the multiplier 0.23 , which in case of crowd with density 0.6 ped. $/ \mathrm{m}^{2}$ may be quite insufficient. Also, [8] explicitly notes that due to the many uncertainties accompanying such a kind of analysis more detailed checks must be done.

Accordingly, the results obtain regarding BS 5400 [5] are, again, based on analysis of the third mode of vibration. The overall results are summarized in Table 3.

Table 3: Maximum accelerations according to [5]

\begin{tabular}{ccccccc}
\hline mode & $f, \mathrm{~Hz}$ & $\psi$ & $K$ & $y_{s}[\mathrm{~m}]$ & $a_{\text {vert }}, \mathrm{m} / \mathrm{s}^{2}$ & $a_{\text {limit }}, \mathrm{m} / \mathrm{s}^{2}$ \\
\hline Mode 3 & 3.02 & 14.06 & 0.88 & 0.00012 & 0.53 & 0.87 \\
\hline
\end{tabular}

The result of $a_{\mathrm{vert}}=0.53 \mathrm{~m} / \mathrm{s}^{2}$ is in compliance with the imposed limit of $a_{\text {limit }}=0.87 \mathrm{~m} / \mathrm{s}^{2}$, though it should be emphasized that this procedure does not mention anything about crowd behaviour or structural vibrations due to a certain number of pedestrians. The result acquired is very close the one obtained through [4] due to impact of one person $-a_{\text {vert }, 1}=0.58 \mathrm{~m} / \mathrm{s}^{2}$.

\subsubsection{DYNAMIC RESPONSE CALCULATIONS ACCORDING TO SÉTRA AND HUMAN- INDUCED VIBRATIONS OF FOOTBRIDGES}

Modes from 2 to 5 are considered as relevant regarding dynamic analysis. All of these modes are torsional except for the 3-rd one which corresponds to the first bending mode with $f_{3}=3.02 \mathrm{~Hz}$.

As the procedures in Sétra and Human-Induced Vibrations of Footbridges (HIVOSS) assume that the bridge deck will be loaded by a crowd with a certain density, the 
modal mass should be altered for each traffic/bridge class and thereby resulting in variation of the natural mode shapes and natural frequencies (Figure 4 and Table 1).

Tables $4-6$ exhibit the results for the three bridge classes with densities 0.5 ped. $/ \mathrm{m}^{2}, 0.8$ ped. $/ \mathrm{m}^{2}$ and 1.0 ped. $/ \mathrm{m}^{2}$. The highest vibration levels for density $d=$ 0.5 ped. $/ \mathrm{m}^{2}$ are calculated in the third mode of vibration $-a_{\text {vert }}=0.57 \mathrm{~m} / \mathrm{s}^{2}$. This is understandable since the third mode is a bending one, characterized by the low modal damping ratio and modal mass. The response in this mode when the crowd density is $d=0.8$ ped. $/ \mathrm{m}^{2}$ is lower due to the decrease in the reduction factor. On the other hand, the response when the crowd density is $d=1.0$ ped. $/ \mathrm{m}^{2}$, is significantly increased, since the number of pedestrians is higher compared to the other two bridge classes. To sum up, the most susceptible, considering this regulation, is the third

Table 4: Vertical accelerations for all relevant modes due to crowd with density $d=$ 0.5 ped. $/ \mathrm{m}^{2}$ according to Sétra [6]

\begin{tabular}{cccccc}
\hline Mode & $f, \mathrm{~Hz}$ & $\psi$ & $P_{\text {vert }}, \mathrm{kN}$ & $F_{\text {modal,vert }}, \mathrm{kN}$ & $a_{\text {vert }}, \mathrm{m} / \mathrm{s}^{2}$ \\
\hline Mode 2 & 1.64 & 0.91 & 16.02 & 2.67 & 0.15 \\
Mode 3 & 2.85 & 0.31 & 0.42 & 0.34 & 0.57 \\
Mode 4 & 3.21 & 0.76 & 2.88 & 1.06 & 0.16 \\
Mode 5 & 3.29 & 0.86 & 4.69 & 0.66 & 0.02 \\
\hline
\end{tabular}

Table 5: Vertical accelerations for all relevant modes due to crowd with density $d=$ $0.8 \mathrm{ped} . / \mathrm{m}^{2}$ according to Sétra [6]

\begin{tabular}{cccccc}
\hline Mode & $f, \mathrm{~Hz}$ & $\psi$ & $P_{\text {vert }}, \mathrm{kN}$ & $F_{\text {modal, } \text { vert }}, \mathrm{kN}$ & $a_{\text {vert }}, \mathrm{m} / \mathrm{s}^{2}$ \\
\hline Mode 2 & 1.6 & 0.86 & 19.03 & 3.08 & 0.17 \\
Mode 3 & 2.76 & 0.20 & 0.35 & 0.27 & 0.45 \\
Mode 4 & 3.15 & 0.69 & 3.32 & 1.21 & 0.19 \\
Mode 5 & 3.21 & 0.77 & 5.26 & 0.72 & 0.02 \\
\hline
\end{tabular}

Table 6: Vertical accelerations for all relevant modes due to crowd with density $d=$ 1.0 ped. $/ \mathrm{m}^{2}$ according to Sétra [6]

\begin{tabular}{cccccc}
\hline Mode & $f, \mathrm{~Hz}$ & $\psi$ & $P_{\text {vert }}, \mathrm{kN}$ & $F_{\text {modal,vert }}, \mathrm{kN}$ & $a_{\text {vert }}, \mathrm{m} / \mathrm{s}^{2}$ \\
\hline Mode 2 & 1.58 & 0.82 & 25.1 & 3.99 & 0.22 \\
Mode 3 & 2.71 & 0.14 & 1.04 & 0.78 & 1.27 \\
Mode 4 & 3.12 & 0.65 & 4.95 & 1.78 & 0.27 \\
Mode 5 & 3.16 & 0.70 & 5.37 & 0.72 & 0.02 \\
\hline
\end{tabular}


mode of vibrations with calculated maximum accelerations of $a_{\mathrm{vert}}=1.27 \mathrm{~m} / \mathrm{s}^{2}$.

The same reasoning could be applied for Tables $7-11$, showing the results for the five traffic classes considered in [7]. Similarly, the most significant reduction for the $\psi$-factor values is in the third mode. Nevertheless, the responses in this mode are obviously the maximum ones for all traffic classes, reaching value of $2.17 \mathrm{~m} / \mathrm{s}^{2}$ for crowd density of 1.0 ped. $/ \mathrm{m}^{2}$. Explanation for that could be found agian in the perceptibly smaller damping ratio for this mode compared with the other three modes (Fig.3). Contrary to the simple proportion rules, the response due to crowd with $d=1.0$ ped. $/ \mathrm{m}^{2}$ is twice as big as the response due to crowd with $d=1.5$ ped. $/ \mathrm{m}^{2}$ - this comes from the alteration in the natural frequency, which leads to a smaller reduction factor for the case of the higher density.

Table 7: Vertical accelerations for all relevant modes due to crowd with density $d=$ 15 ped. [7]

\begin{tabular}{cccccc}
\hline Mode & $f, \mathrm{~Hz}$ & $\psi$ & $P_{\text {vert }}, \mathrm{kN}$ & $F_{\text {modal,vert }}, \mathrm{kN}$ & $a_{\text {vert }}, \mathrm{m} / \mathrm{s}^{2}$ \\
\hline Mode 2 & 1.70 & 1.00 & 5.67 & 0.99 & 0.06 \\
Mode 3 & 3.00 & 0.14 & 0.24 & 0.20 & 0.36 \\
Mode 4 & 3.29 & 0.22 & 1.08 & 0.41 & 0.06 \\
Mode 5 & 3.42 & 0.25 & 1.75 & 0.26 & 0.01 \\
\hline
\end{tabular}

Table 8: Vertical accelerations for all relevant modes due to crowd with density $d=$ 0.2 ped. $/ \mathrm{m}^{2}$ [7]

\begin{tabular}{cccccc}
\hline Mode & $f, \mathrm{~Hz}$ & $\psi$ & $P_{\text {vert }}, \mathrm{kN}$ & $F_{\text {modal,vert }}, \mathrm{kN}$ & $a_{\text {vert }}, \mathrm{m} / \mathrm{s}^{2}$ \\
\hline Mode 2 & 1.68 & 0.96 & 10.62 & 1.83 & 0.11 \\
Mode 3 & 2.95 & 0.12 & 0.43 & 0.35 & 0.61 \\
Mode 4 & 3.26 & 0.21 & 2.04 & 0.76 & 0.12 \\
Mode 5 & 3.37 & 0.24 & 3.34 & 0.49 & 0.01 \\
\hline
\end{tabular}

Table 9: Vertical accelerations for all relevant modes due to crowd with density $d=$ 0.5 ped. $/ \mathrm{m}^{2}$ [7]

\begin{tabular}{cccccc}
\hline Mode & $f, \mathrm{~Hz}$ & $\psi$ & $P_{\text {vert }}, \mathrm{kN}$ & $F_{\text {modal,vert }}, \mathrm{kN}$ & $a_{\text {vert }}, \mathrm{m} / \mathrm{s}^{2}$ \\
\hline Mode 2 & 1.64 & 0.86 & 15.17 & 2.53 & 0.15 \\
Mode 3 & 2.85 & 0.10 & 0.53 & 0.42 & 0.71 \\
Mode 4 & 3.21 & 0.20 & 2.98 & 1.10 & 0.17 \\
Mode 5 & 3.29 & 0.22 & 4.77 & 0.67 & 0.02 \\
\hline
\end{tabular}


Table 10: Vertical accelerations for all relevant modes due to crowd with density $d=1.0$ ped. $/ \mathrm{m}^{2}[7]$

\begin{tabular}{cccccc}
\hline Mode & $f, \mathrm{~Hz}$ & $\psi$ & $P_{\text {vert }}, \mathrm{kN}$ & $F_{\text {modal,vert }}, \mathrm{kN}$ & $a_{\text {vert }}, \mathrm{m} / \mathrm{s}^{2}$ \\
\hline Mode 2 & 1.58 & 0.72 & 22.09 & 3.51 & 0.19 \\
Mode 3 & 2.71 & 0.06 & 1.77 & 1.33 & 2.17 \\
Mode 4 & 3.12 & 0.17 & 5.24 & 1.89 & 0.29 \\
Mode 5 & 3.16 & 0.18 & 5.62 & 0.75 & 0.02 \\
\hline
\end{tabular}

Table 11: Vertical accelerations for all relevant modes due to crowd with density $d=1.5$ ped. $/ \mathrm{m}^{2}[7]$

\begin{tabular}{cccccc}
\hline Mode & $f, \mathrm{~Hz}$ & $\psi$ & $P_{\text {vert }}, \mathrm{kN}$ & $F_{\text {modal,vert }}, \mathrm{kN}$ & $a_{\text {vert }}, \mathrm{m} / \mathrm{s}^{2}$ \\
\hline Mode 2 & 1.52 & 0.60 & 22.34 & 3.40 & 0.18 \\
Mode 3 & 2.59 & 0.02 & 0.90 & 0.65 & 1.01 \\
Mode 4 & 3.04 & 0.15 & 5.58 & 1.97 & 0.29 \\
Mode 5 & 3.05 & 0.15 & 5.69 & 0.72 & 0.02 \\
\hline
\end{tabular}

The analysis conducted according the two guidelines shows that the structure will possibly experience vibration levels exceeding the limit of medium comfort of $a_{\text {medium }}=1.0 \mathrm{~m} / \mathrm{s}^{2}$ (Fig. 5). A simple visual inspection shows that the results agree relatively well for the crowd density $0.5 \mathrm{ped} . / \mathrm{m}^{2}$ for all of the four modes considered. The results for the other common density for the two regulations $-d=1$ ped. $/ \mathrm{m}^{2}-$
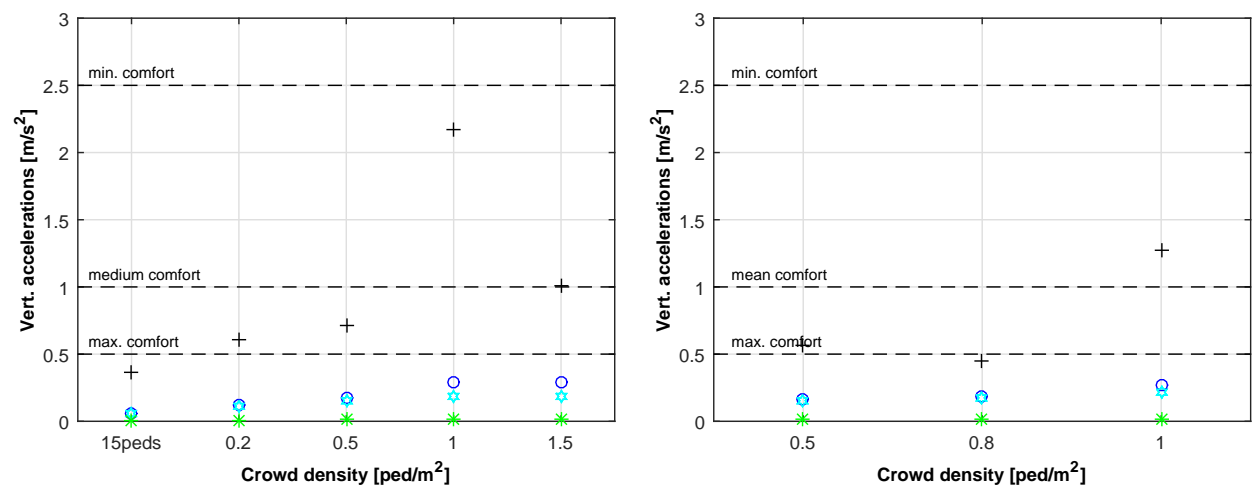

Fig. 5: Values of the vertical accelerations according to HIVOSS (left) and Sétra (right): blue hex - Mode 2; black + - Mode 3; blue o-Mode 4; green * - Mode 5 . 
cohere well, except for the 3-rd one, for which the value acquired by [7] is approximately $2.17 \mathrm{~m} / \mathrm{s}^{2}$, whereas [6] gives value of $1.27 \mathrm{~m} / \mathrm{s}^{2}$ for this mode of vibration. The reason for such a discrepancy of almost $50 \%$ is implicated in the reduction factor $\psi$. The following conclusion can be drawn based on the graph (Fig. 6): for crowd densities up to 0.8 ped. $/ \mathrm{m}^{2}$ medium comfort level is provided; for higher densities medium level of comfort is insured for $1.5 \mathrm{ped} . / \mathrm{m}^{2}$, having in mind that the value of calculated accelerations is on the very limit for mode 3: $a_{d=1.5, \text { Mode } 3}=1.01 \mathrm{~m} / \mathrm{s}^{2}$; for density $d=1.0 \mathrm{~m} / \mathrm{s}^{2}$, both guides give value above the medium comfort level, where the value acquired through HIVOSS is even close the limit value for minimal comfort.

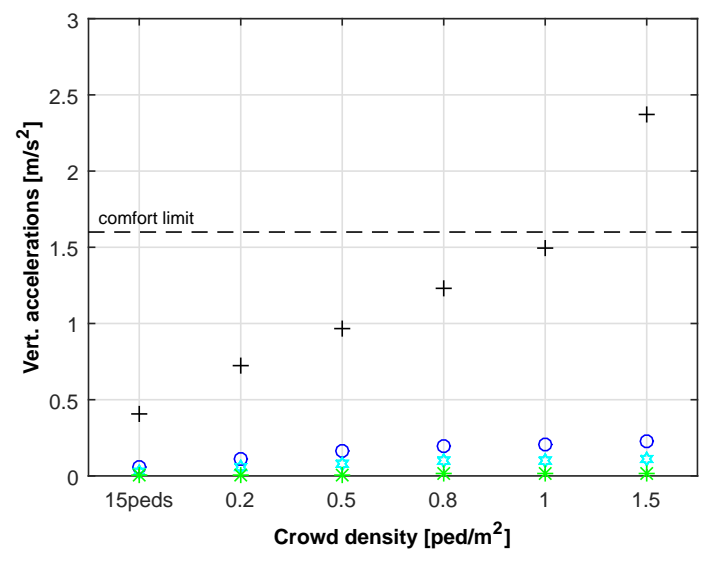

Fig. 6: Values of the vertical accelerations according to UK National Annex to Eurocode 1: blue hex - Mode 2; black + - Mode 3; blue o - Mode 4; green * - Mode 5.

\subsubsection{DYNAMIC RESPONSE CALCULATIONS ACCORDING TO UK NATIONAL ANNEX TO EUROCODE 1}

Tables 12 - 17 present the calculation performed according to [9]. Although the standard gives several reference crowd densities, it is underlined that they are recommendable. Consequently, the crowd densities regarded here coincide with the densities used in [6] and [7], thereby allowing for direct comparison in between.

The results obtained here show that Mode 3 is the dominant one. The responses in Mode 3 are the highest for all bridge classes because of the very low damping ratio, although the reduction factor and respectively the vertical modal load for this mode are very small. The acceleration levels increase gradually from the case with 
Table 12: Vertical accelerations for all relevant modes due to crowd of $d=15$ ped. [9]

\begin{tabular}{ccccccc}
\hline Mode & $f, \mathrm{~Hz}$ & $\gamma$ & $k\left(f_{n}\right)$ & $P_{\text {vert }}, \mathrm{kN}$ & $F_{\text {modal,vert }}, \mathrm{kN}$ & $a_{\text {vert }}, \mathrm{m} / \mathrm{s}^{2}$ \\
\hline Mode 2 & 1.7 & 0.14 & 0.95 & 2.99 & 0.52 & 0.03 \\
Mode 3 & 3 & 0.01 & 0.28 & 0.27 & 0.23 & 0.41 \\
Mode 4 & 3.29 & 0.1 & 0.36 & 0.97 & 0.36 & 0.06 \\
Mode 5 & 3.42 & 0.21 & 0.37 & 1.44 & 0.22 & 0.01 \\
\hline
\end{tabular}

Table 13: Vertical accelerations for all relevant modes due to crowd with density $d=0.2$ ped. $/ \mathrm{m}^{2}[9]$

\begin{tabular}{ccccccc}
\hline Mode & $f, \mathrm{~Hz}$ & $\gamma$ & $k\left(f_{n}\right)$ & $P_{\text {vert }}, \mathrm{kN}$ & $F_{\text {modal, vert }}, \mathrm{kN}$ & $a_{\text {vert }}, \mathrm{m} / \mathrm{s}^{2}$ \\
\hline Mode 2 & 1.68 & 0.14 & 0.93 & 5.78 & 0.99 & 0.06 \\
Mode 3 & 2.95 & 0.01 & 0.26 & 0.5 & 0.41 & 0.72 \\
Mode 4 & 3.26 & 0.1 & 0.35 & 1.87 & 0.7 & 0.11 \\
Mode 5 & 3.37 & 0.21 & 0.37 & 2.8 & 0.41 & 0.01 \\
\hline
\end{tabular}

Table 14: Vertical accelerations for all relevant modes due to crowd with density $d=0.5$ ped. $/ \mathrm{m}^{2}[9]$

\begin{tabular}{ccccccc}
\hline Mode & $f, \mathrm{~Hz}$ & $\gamma$ & $k\left(f_{n}\right)$ & $P_{\text {vert }}, \mathrm{kN}$ & $F_{\text {modal,vert }}, \mathrm{kN}$ & $a_{\text {vert }}, \mathrm{m} / \mathrm{s}^{2}$ \\
\hline Mode 2 & 1.64 & 0.14 & 0.91 & 8.85 & 1.48 & 0.08 \\
Mode 3 & 2.85 & 0.01 & 0.24 & 0.72 & 0.57 & 0.97 \\
Mode 4 & 3.21 & 0.1 & 0.34 & 2.87 & 1.06 & 0.16 \\
Mode 5 & 3.29 & 0.21 & 0.35 & 4.29 & 0.61 & 0.01 \\
\hline
\end{tabular}

Table 15: Vertical accelerations for all relevant modes due to crowd with density $d=0.8$ ped. $/ \mathrm{m}^{2}[9]$

\begin{tabular}{ccccccc}
\hline Mode & $f, \mathrm{~Hz}$ & $\gamma$ & $k\left(f_{n}\right)$ & $P_{\text {vert }}, \mathrm{kN}$ & $F_{\text {modal, vert }}, \mathrm{kN}$ & $a_{\text {vert }}, \mathrm{m} / \mathrm{s}^{2}$ \\
\hline Mode 2 & 1.6 & 0.14 & 0.88 & 10.82 & 1.75 & 0.1 \\
Mode 3 & 2.76 & 0.01 & 0.25 & 0.97 & 0.74 & 1.23 \\
Mode 4 & 3.15 & 0.1 & 0.33 & 3.49 & 1.27 & 0.2 \\
Mode 5 & 3.21 & 0.21 & 0.34 & 5.21 & 0.71 & 0.02 \\
\hline
\end{tabular}

15 pedestrians $-a=0.41 \mathrm{~m} / \mathrm{s}^{2}-$ to the highest density of $d=1.5$ ped. $/ \mathrm{m}^{2}-a=$ $2.37 \mathrm{~m} / \mathrm{s}^{2}$. For the latter particular case the comfort criteria is not met $-a_{\text {limit,max }}=$ $1.6 \mathrm{~m} / \mathrm{s}^{2}$. 
Table 16: Vertical accelerations for all relevant modes due to crowd with density $d=1.0$ ped. $/ \mathrm{m}^{2}[9]$

\begin{tabular}{ccccccc}
\hline Mode & $f, \mathrm{~Hz}$ & $\gamma$ & $k\left(f_{n}\right)$ & $P_{\text {vert }}, \mathrm{kN}$ & $F_{\text {modal, vert }}, \mathrm{kN}$ & $a_{\text {vert }}, \mathrm{m} / \mathrm{s}^{2}$ \\
\hline Mode 2 & 1.58 & 0.14 & 0.86 & 11.81 & 1.88 & 0.1 \\
Mode 3 & 2.71 & 0.01 & 0.28 & 1.22 & 0.91 & 1.49 \\
Mode 4 & 3.12 & 0.1 & 0.32 & 3.8 & 1.37 & 0.21 \\
Mode 5 & 3.16 & 0.21 & 0.33 & 5.63 & 0.75 & 0.02 \\
\hline
\end{tabular}

Table 17: Vertical accelerations for all relevant modes due to crowd with density $d=1.5$ ped. $/ \mathrm{m}^{2}[9]$

\begin{tabular}{ccccccc}
\hline Mode & $f, \mathrm{~Hz}$ & $\gamma$ & $k\left(f_{n}\right)$ & $P_{\text {vert }}, \mathrm{kN}$ & $F_{\text {modal, vert }}, \mathrm{kN}$ & $a_{\text {vert }}, \mathrm{m} / \mathrm{s}^{2}$ \\
\hline Mode 2 & 1.52 & 0.14 & 0.8 & 13.58 & 2.07 & 0.11 \\
Mode 3 & 2.59 & 0.01 & 0.4 & 2.12 & 1.52 & 2.37 \\
Mode 4 & 3.04 & 0.1 & 0.29 & 4.29 & 1.51 & 0.23 \\
Mode 5 & 3.05 & 0.21 & 0.3 & 6.22 & 0.79 & 0.02 \\
\hline
\end{tabular}

Regarding the comfort criteria, it is worth mentioning that the bridge is situated in a rural area and it is elevated over the ground level on $5.5 \mathrm{~m}$. The comfort limit for the Eeklo footbridge could be assumed as $a_{\text {limit }}=1.6 \mathrm{~m} / \mathrm{s}^{2}([3,9])$ assuming the relevant coefficients according to Table 18 . This simply means that the comfort level is insured only for bridge classes with density up to $d=1.0 \mathrm{ped} . / \mathrm{m}^{2}$.

Table 18: Coefficients for determining the comfort critiria according to [9]

\begin{tabular}{llll}
\hline Rural environment & $k_{1}=1.6$ & Primary route & $k_{2}=1.0$ \\
Bridge height from $4 \mathrm{~m}$ to $8 \mathrm{~m}$ & $k_{3}=1.0$ & Exposure factor & $k_{4}=1.0$ \\
\hline
\end{tabular}

\section{Conclusions}

A comprehensive exploration of the current codes of practice accounting for either single pedestrian loading and crowd loading, and assessing the dynamic behaviour of pedestrian structures is elaborated. The analysis conducted in Section 3 shows not only differences, but major discrepancies between the results obtained by the different regulations.

The detailed procedures for analyzing crowd loading provided by Sétra [6] and HIVOSS [7] overlap in most assumptions. Nevertheless, due to slight differences in the reduction factor definition, the results for common crowd densities are not in a 
good conformity, but differ with some admissible margin. The overall results show admissible values, in compliance with the limits imposed by the respective regulations. With small exceptions, the acceleration levels obtained satisfy the medium comfort criteria levels. Though, a significant dispersion in the results is present due to some differences in the made assumptions. Although coinciding in most premises adopted, the procedures in [6] and [7] show different vertical acceleration levels due to differences in the governing reduction factor $\psi$. The analysis according to UK National annex is extrapolated for all crowd densities regarded in Sétra and HIVOSS. For low crowd densities up to $d=0.5$ ped. $/ \mathrm{m}^{2}$ the results according to UK National annex and HIVOSS agree very well. For the higher densities, there is perceptible difference in the results derived by UK National annex and Sétra and HIVOSS. Common implication is the dominating response in Mode 3, which is stemming from the low modal damping ratio.

\section{REFERENCES}

[1] L. ZDRAVKOV (2012) Specific features in design in footbridges, located between roofs of aboveground steel tanks. In: Proceeding of 12th International Scientific Conference VSU'2012, Sofia, Bulgaria.

[2] KR. Kasapova (2018) Human-induced vibrations on footbridges and the impact of human-human interaction. PhD thesis, UACEG, So

a, Bulgaria.

[3] Kr. Kasapova, D. Dinev (2020) Human-induced vibrations on footbridges. current codes of practice - overview. Journal of Theoretical and Applied Mechanics 50 142157.

[4] European committee For Standardization (2004) Eurocode 5: Design of timber structures - Part 2: Bridges.

[5] BRITish STANDARD (1978) BS 5400: Steel, concrete and composite bridges (Part 2: Specification for loads).

[6] Association Française de Génie Civil, Sétra/AFGC (2006) Evaluation du comportement vibratoire des passerelles piétonnes sous l'action des piétons. Sétra, Paris.

[7] HIVOSS (2008) "Design of Footbridges". Research Fund for Coal and Steel.

[8] EuropeAn COMMITTEE FOR STANDARDIZATION (2005) Eurocode 0: Basis of structural design.

[9] British Standard (2003) BS NA EN 1991-2: UK National Annex to Eurocode 1. Actions on structures. Traffic loads on bridges. 